Article

\title{
Biomass Fuel and Combustion Conditions Selection in a Fixed Bed Combustor
}

\author{
María E. Arce ${ }^{1{ }^{*} *}$, Ángeles Saavedra ${ }^{2}$, José L. Míguez ${ }^{1}$, Enrique Granada ${ }^{1}$ and Antón Cacabelos ${ }^{1}$ \\ 1 ETS Ingenieros Industriales, University of Vigo, Lagoas-Marcosende s/n, Vigo 36310, Spain; \\ E-Mails: jmiguez@uvigo.es (J.L.M.); egranada@uvigo.es (E.G.); acacabelos@uvigo.es (A.C.) \\ 2 ETS Ingeniería de Minas, University of Vigo, Lagoas-Marcosende s/n, Vigo 36310, Spain; \\ E-Mail: saavedra@uvigo.es
}

* Author to whom correspondence should be addressed; E-Mail: elena.arce@uvigo.es; Tel.: +34-986-804-939.

Received: 27 September 2013; in revised form: 4 November 2013 / Accepted: 6 November 2013 / Published: 18 November 2013

\begin{abstract}
The biomass market has experienced an increase in development, leading to research and development efforts that are focused on determining optimal biofuel combustion conditions. Biomass combustion is a complex process that involves divergent parameters and thus requires the use of advanced analysis methods. This study proposes combining grey relational analysis (GRA) and error propagation theory (EPT) to select a biofuel and its optimal combustion conditions. This research will study three biofuels that are currently used in a region of South Europe (Spain), and the most important variables that affect combustion are the ignition front propagation speed and the highest temperature that is reached at the fixed bed combustor. The results demonstrate that a combination of both theories for the analysis of solid-state thermochemical phenomena enables a fast and simple way of choosing the best configuration for each fuel.
\end{abstract}

Keywords: grey relational grade error (GRGe); fuel behavior; fixed-bed combustion error propagation theory (EPT); grey relational analysis (GRA)

\section{Nomenclature:}

$\begin{array}{ll}\mathrm{AE} & \text { air excess } \\ \mathrm{AMF} & \text { air mass flow }\left(\mathrm{kg} \mathrm{m}^{-2} \mathrm{~s}^{-1}\right) \\ A_{p} & \text { surface area of the characteristic particle }\left(\mathrm{m}^{2}\right) \\ \mathrm{bp} & \text { brassica pellet }\end{array}$




$\begin{array}{ll}E r & \text { radius of the equivalent sphere } \\ \text { EPT } & \text { error propagation theory } \\ \text { GRA } & \text { grey relational analysis } \\ \text { GRC } & \text { grey relational coefficient }(\xi) \\ \text { GRG } & \text { grey relational grade }(\gamma) \\ \text { GRGe } & \text { grey relational grade error }(\Delta(\gamma)) \\ \text { IMF } & \text { ignition mass flux }\left(\mathrm{kg} \mathrm{m}^{-2} \mathrm{~s}^{-1}\right) \\ \text { IMFU } & \text { ignition mass flux uncertainty }\left(\mathrm{kg} \mathrm{m}^{-2} \mathrm{~s}^{-1}\right) \\ k & \text { form factor of the Rosin-Ramler distribution }(-) \\ \text { LHV } & \text { lower heating value in water basis with ash }\left(\text { as received) }\left(\mathrm{kJ} \mathrm{kg}^{-1}\right)\right. \\ M & \text { mass }(\mathrm{kg}) \\ m_{I G}^{\prime \prime} & \text { ignition mass flux }\left(\mathrm{kg} \mathrm{m}{ }^{-2} \mathrm{~s}^{-1}\right) \\ \text { MT } & \text { maximum temperature }\left({ }^{\circ} \mathrm{C}\right) \\ M_{T} & \text { Total mass of the distribution }(\mathrm{kg}) \\ \text { MTU } & \text { maximum temperature uncertainty }\left({ }^{\circ} \mathrm{C}\right) \\ \text { os } & \text { olive stone } \\ S_{v o l} & \text { sphere volume }\left(\mathrm{m}^{3}\right) \\ V_{p} & \text { volume of the characteristic particle }\left(\mathrm{m}^{3}\right) \\ \text { wp } & \text { wood pellet } \\ \zeta & \text { distinguish coefficient } \\ \lambda & \text { characteristic length of the distribution }(\mathrm{m}) \\ \mu & \text { characteristic length of the distribution }(\mathrm{kg}) \\ \psi & \text { sphericity }\end{array}$

\section{Introduction}

Research and investments have been directed into renewable energies due to the growing renewable energy demand that is supported by state plans. Biomass has played a large role in these plans, mainly due to its $\mathrm{CO}_{2}$ neutrality and high availability. Currently, biomass is, in absolute terms, Spain's most important renewable energy source, accounting for 30\% of primary renewable energy production [1]. This trend extends to the whole European Union, and it is expected that the direct use of biomass in the residential sector will double by 2020 [2].

Of the different thermochemical conversion techniques, combustion is the most extensively used and developed technique for the utilization of biomass [3]. Thus, to increase combustion development, the whole process needs to be optimized in terms of efficiency and cost. Because of this necessity, developing research has focused on three main aspects: the fuel properties, boiler characteristics, and emissions [4-10].

An important issue that is related to the utilization of biomass as an energy resource is that biomass has a disperse origin. Due to this fact, and to reduce logistic costs, most operations occur in small-scale plants [11]. Of all the biomass low-output boiler combustion methods, fixed bed boilers are most 
desirable because of their simplicity, reduced costs, higher automation, and batch control, which, in turn, enable the feeding of heterogeneous biofuels [12].

However, studies regarding the behavior and selection of biomass are important for biofuel combustion research. Numerous parameters influence the combustion process complexity, thus preventing the use of unique criteria for biofuel selection and constraining research to a reduced number of variables. The fundamental variables that have been identified for biofuel behavior analysis are the ignition mass flow (fuel burn rate) and the maximum bed temperature $[13,14]$. Both of these variables depend on the supplied air mass flow rate to the bed. The analysis and quantification of each variable become difficult due to the inability to separate out the variables and parameters that affect combustion and to the presence of divergences among them.

Grey relational analysis (GRA) is an adequate tool for solving problems that comprise numerous interrelated parameters and variables [15]. The ability to apply this analysis to a set of limited data without the need for a minimum amount of data is an advantage over other methods [16]. Moreover, GRA enables the processing of variables with different attributes, scales, and units. Thus, this method is more versatile than other decision tools [17]. Another characteristic of GRA is the ability to work with variables that are optimized in opposite directions [18]. For the case that is explored here, the objective is to select a fuel that provides the highest mass flow with the lowest maximum temperature in order to avoid problems that are related to slag formation after ash smelting [19].

However, GRA does not take into account the uncertainty that is associated with the initial measurement sequences and how this uncertainty propagates along the GRA calculation steps [20]. As in the case of The Joint Committee for Guides in Metrolog [21], a measurement result is an estimation of the magnitude of a value and must be determined along with the uncertainty that is related to the measurement process.

This study proposes combining GRA and error propagation theory (EPT), based on a recommendation from [20], to study three biomass fuels to estimate the optimal combustion parameters and to identify the main factors that affect the propagation speed and the maximum temperature that is reached by each fuel.

\section{Experimental Section and Methodology}

\subsection{Experimental Plant}

The experimental plant and data analysis that were used in this study are based on previous research results that were reported by [22]. The experimental plant design (Figures 1 and 2) was completed by taking into account the results of other studies that were performed on fixed bed combustion equipment. The experimental plant comprises two modules: the first module, called the working module, is located on the burner, and the second module, the feeding and control module, is located on the fan. Both structures are connected by an air duct, and a signal cable system joins the thermocouples to the data acquisition card. This configuration satisfies the need to perform the experiments outside without requiring the relocation of the feeding and control module. The experiments were performed outside because of the impracticality of conduct the tests in the facilities. 
Figure 1. Working module computer-aided design (CAD) scheme.

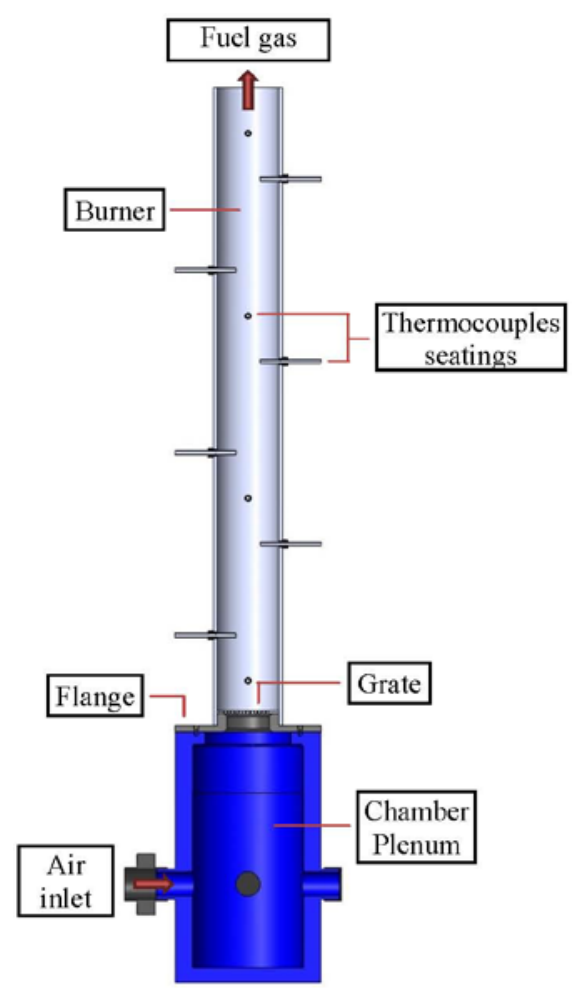

Figure 2. Power and control module.

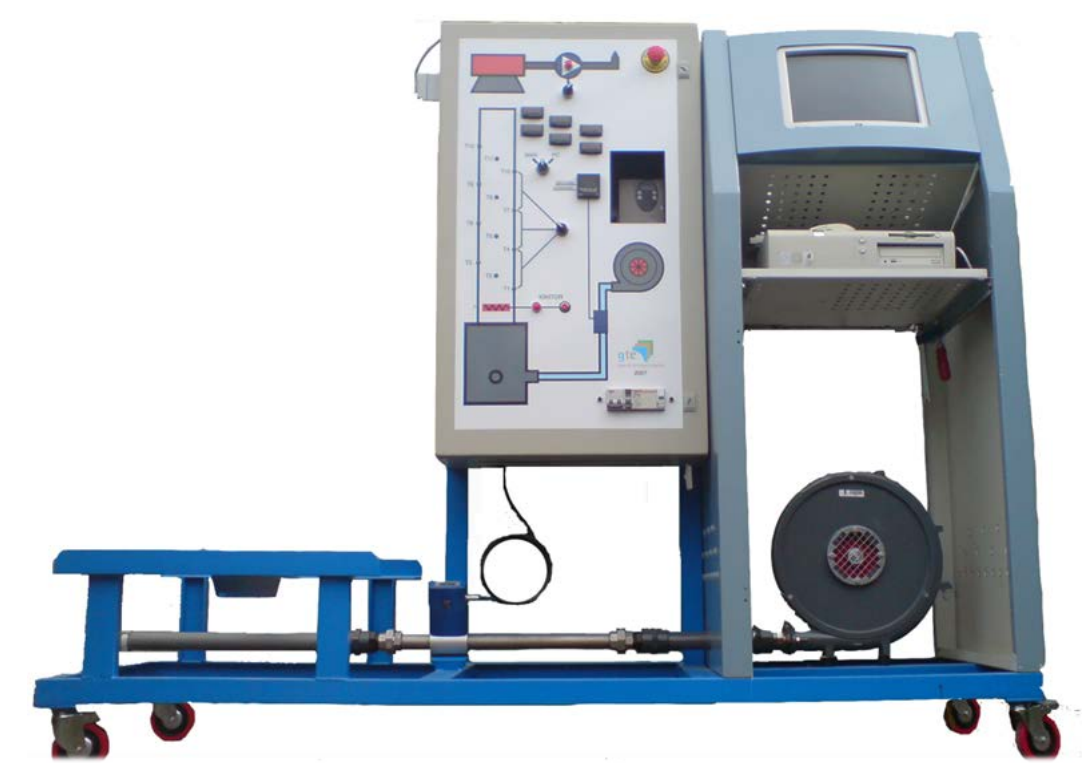

As shown in Figure 1, the burner is a cylindrical tube with $0.135 \mathrm{~m}$ in internal diameter and $0.007 \mathrm{~m}$ in thickness that is made of low-alloy steel. An axisymmetrical tube was used to attain both a simple geometry and minimize unexpected fringe effects on the walls of the tube. To avoid the surface effects that are due to the forced particle arrangement, the tube diameter is 20 times the size of the particles that will be tested. Based on experimental results, the estimated ignition front propagation speed and temperature as a function of the inlet air mass flow rate indicate that, for a medium-low calorific value and the selected tube diameter, a thermal power similar to that of a low-power domestic boiler can be attained [23]. 
Because one of the purposes of the tube was to operate as a chimney by expelling the combustion gases, a total effective tube height of $1 \mathrm{~m}$ was chosen. To measure the ignition front propagation speed and the fixed bed temperature, a total of 12 type- $\mathrm{K}$ thermocouples $(\mathrm{Ni}-\mathrm{Cr} / \mathrm{Ni}-\mathrm{Al})$ were helicoidally positioned along the first $0.7 \mathrm{~m}$ of the tube length. Each thermocouple was inserted using a bulkhead connector.

The selection of type-K thermocouples was justified by two reasons. The first and main reason is the previous results that were provided by other authors, i.e., the maximum fixed bed temperature is approximately $1300{ }^{\circ} \mathrm{C}[14,24,25]$; because type-K thermocouples work up to $1372{ }^{\circ} \mathrm{C}$, these thermocouples are suitable for the aforementioned application. The type-K thermocouples had $150 \mathrm{~mm}$ stems because the tips had to reach into the fixed bed as far as the cylinder axis to minimize fringe effects.

The ignition front propagation speed is determined by measuring the time that is needed to attain a predetermined temperature between two adjacent thermocouples. The distance between thermocouples is a known measurement and includes the associated uncertainty.

To protect a thermocouple from high temperatures, it is removed as soon as the ignition front surpasses the thermocouple level. Once the thermocouple is removed, it is replaced by a manual probe to measure the maximum temperature that is reached by the ignition front. The manual probe does not use the same transducer. Thus, the manual probe is limited not only by the maximum peak temperature of $1372{ }^{\circ} \mathrm{C}$, but also by the maximum $1000^{\circ} \mathrm{C}$ of the transducer.

The thermocouples were not lined up to avoid any possible interference inside the fixed bed. It should be noted that, although there are twelve thermocouples along the tube, not all of the measurements were used at the same time for a particular experiment. Data from the first and last thermocouple measurements are dismissed. In the case of the first measurements, this omission is related to the start-up of the initial phase, during which the system is in a non-steady state; in the case of the last measurements, this omission is related to the fact that the thermocouples are at the shutdown phase and are on the brink of developing resonance phenomena [23].

The air feeding system comprises a centrifugal fan and a flow meter. The air mass flow penetrates through an opening on the fixed bed. The plant was designed with a feed bypass to avoid problems with recirculation and to ensure a completely developed turbulent flow at the tube entrance.

\subsection{Ignition Front Propagation in a Fixed Bed}

The combustor works as a batch reactor. During the experiment, the combustion is started by manual ignition at the top layer of the fuel, and the ignition propagation front advances downwards. Considering the air direction, the system is in a counter-current configuration [26]. In counter-current combustion models, the radiation (gas-particle and particle-particle) and heat-transfer (particle-particle) cause the ignition to propagate in the opposite direction of the air flow; however, convection complicates the propagation due to bed cooling [27].

The fixed bed is stratified during the combustion process, enabling the identification of the phases that the solid fuel exhibits when it thermally degrades in the presence of oxygen (Figure 3 ).

A gas-char reaction phase occurs during the combustion of fixed carbon (the ignition zone). Most of the bed energy is generated in this layer. The heat that is produced by the combustion of volatiles and by fixed carbon oxidation propagates downwards through the bed due to gas-particle and particle-particle 
radiation and to particle-particle heat conduction [26]. The incoming cold air from the tube base cools the low-layer particles and absorbs heat in the upward direction, thus hampering heat transfer. The reaction phases depend on the bed layer temperature [9]. If the layer temperature lies between room temperature and $100{ }^{\circ} \mathrm{C}$, the particles are considered to only release water (the drying phase). During the devolatilisation phase, which occurs between $100{ }^{\circ} \mathrm{C}$ and $500{ }^{\circ} \mathrm{C}$, there is a thermal decomposition of the fuel, and maximum temperatures are reached in the ignition front [28]. Although there is no combustion during the drying and devolatilisation phases, the material changes as a result of the high temperature.

Figure 3. Model diagram for ignition front propagation in a fixed bed.

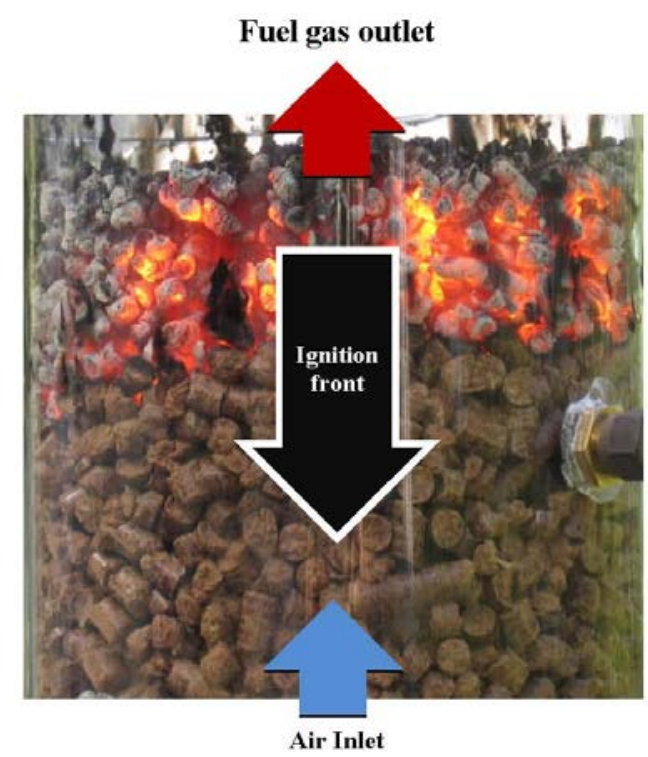

Excess air is used as a parameter to indicate the amount of combusted fuel; therefore, this parameter is related to the ignition front propagation speed. In regions where there is a lack of air (the substoichiometric combustion zone), there is a complete separation of the three phases. In the case of excess air, the phases overlap, as shown in Figure 3. It is difficult to distinguish the different thermochemical processes that the biomass undergoes.

\subsection{Variable Selection}

Generally, a high combustion efficiency is associated with a high calorific value. The highest calorific value of the fuel determines the ignition front speed (the ignition mass flow) [12]. However, high bed temperatures could lead to ash fusion, which, in turn, reduces the energy that is released in the bed [29].

Experiments were conducted using a constant air flow at the bed base. Thus, the variables (the ignition mass flow rate and the maximum temperature) are functions not only of the fuel properties but also of the air mass flow rate, which is a controllable parameter during the combustion process.

It is worth noting that not all fuels tolerate the same air flow rate. This fact results in a lower number of experiments that can be conducted, thus diminishing the sample size. Thus, GRA is suitable because it is applied to diminished systems. 


\subsection{Fuels}

In this study, three different types of biomass were studied, as shown in Figure 4.

Figure 4. Fuel samples that were used in this study. (a) Brassica pellet (bp); (b) Olive stone (os); (c) Wood pellet (wp).

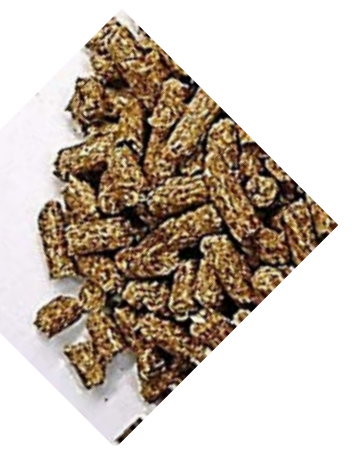

(a)

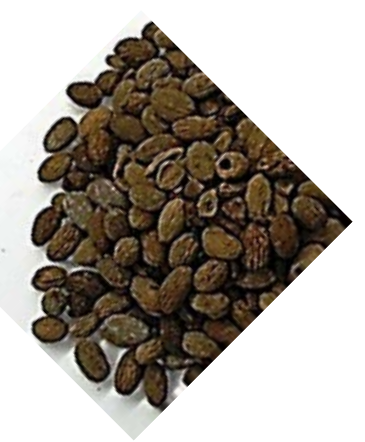

(b)

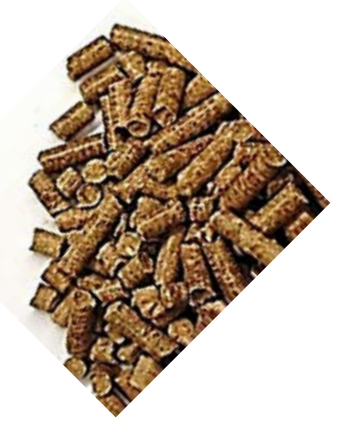

(c)

Their properties are presented in Table 1. The selected types of biomass are abundant and are easily available in Spain. Two of these materials were pellet fuels, a wood mixture and a mixture of energy crops. The third biomass fuel was utilized in its raw form [olive stone (os)].

Table 1. Fuel properties.

\begin{tabular}{cccc}
\hline Fuel type & $\begin{array}{c}\text { Formula dry } \\
\text { basis ash free }\end{array}$ & $\begin{array}{c}\text { Lower heating value } \\
\left(\mathbf{M J ~ k g}^{-1}\right)\end{array}$ & $\begin{array}{c}\text { Water (immediate analysis as } \\
\text { received. Wet basis with ash) (\%) }\end{array}$ \\
\hline Brassica pellet (bp) & $\mathrm{CH}_{1.41} \mathrm{O}_{0.78}$ & 13.1 & 9 \\
Wood pellet (wp) & $\mathrm{CH}_{1.48} \mathrm{O}_{0.71}$ & 16.3 & 6.2 \\
Olive stone (os) & $\mathrm{CH}_{1.50} \mathrm{O}_{0.64}$ & 15.3 & 13 \\
\hline
\end{tabular}

Granulometry

The particles of the fuel materials that were studied, i.e., the solid processed fuels [brassica pellet (bp) and wood pellet (wp)] and the solid unprocessed fuel [olive stone (os)], ranged in size. The material behaviors differ with the heterogeneous geometries, as shown in Figure 5.

Figure 5. Characteristic shape geometry of each fuel.

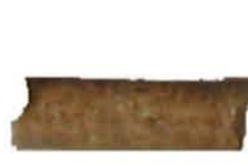

Pellet

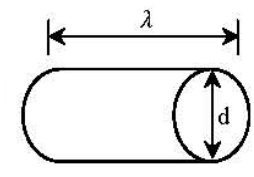

Cilinder

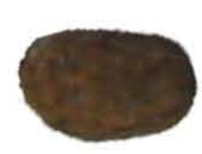

Olive Stone

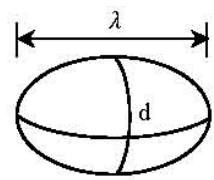

Spheroid

A Rosin-Rammler distribution was used to estimate the shape and size of the characteristic or equivalent particle [30]:

$$
\frac{M(>m)}{M_{T}}=\exp \left[-\left(\frac{m}{\mu}\right)^{\frac{k}{3}}\right]
$$


where:

$\mu \quad$ characteristic mass of the distribution $(\mathrm{kg})$;

$M_{T}$ total mass of the distribution $(\mathrm{kg})$;

$k$ form factor that was used to fit the distribution (-).

Dimensional information was obtained using a MATLAB image shape recognition system. To obtain this information, the particle image contrast was magnified and the geometries were identified using object classification with a Roslin-Rammler distribution function.

The characteristic particle should exclude $1 / e$ of the accumulated total mass $(\bar{Y})$ from the following distribution:

$$
\frac{M(>\lambda)}{M_{T}}=\frac{1}{e}
$$

where:

$\lambda \quad$ characteristic length of the distribution (m).

Once the characteristic particle size of each fuel was determined, the equivalent radius and sphericity were calculated (Table 2):

$$
E r=\left(\frac{3 S_{v o l}}{4 \pi}\right)^{1 / 3}
$$

where:

$S_{\text {vol }} \quad$ sphere volume $\left(\mathrm{m}^{3}\right)$.

$$
\psi=\frac{\pi^{1 / 3}\left(6 V_{p}\right)^{2 / 3}}{A_{p}}
$$

where:

$V_{p} \quad$ volume of the characteristic particle $\left(\mathrm{m}^{3}\right)$;

$A_{p} \quad$ surface area of the characteristic particle $\left(\mathrm{m}^{2}\right)$.

Table 2. Fuel morphology data.

\begin{tabular}{ccc}
\hline Fuel type & Radius of the equivalent sphere $(\boldsymbol{E r})(\mathbf{m m})$ & Sphericity $(\boldsymbol{\psi})$ \\
\hline Brassica pellet (bp) & 4.1 & 0.86 \\
Wood pellet (wp) & 3.8 & 0.87 \\
Olive stone (os) & 4.2 & 0.98 \\
\hline
\end{tabular}

\subsection{Grey Relational Grade Error (GRGe)}

In the following, a procedure is developed to both calculate the GRA and to apply EPT to GRA.

\subsubsection{GRA}

The GRA calculation process can be summarised in the following four steps: 
Step 1. Data normalisation:

- If the highest value is the optimal value (as in the case of the ignition mass flow rate variable):

$$
x_{i, j}=\frac{y_{i, j}-\min _{j} y_{i, j}}{\max _{j} y_{i, j}-\min _{j} y_{i, j}} \in[0,1] ; i=1, \ldots, I ; j=1, \ldots, J
$$

- If the lowest value is the optimal value (as in the case of the maximum temperature variable):

$$
x_{i, j}=\frac{y_{i, j}-\min _{j} y_{i, j}}{\max _{j} y_{i, j}-\min _{j} y_{i, j}} \in[0,1] ; i=1, \ldots, I ; j=1, \ldots, J
$$

where $y_{i}$ represents the variables as a function of the $J$ factors.

The highest normalised values correspond to the best experimental variable values being equal to the ideal value of 1 [31]. The purpose of this step is to transform the input data into comparable sequences that are independent of the units or measurement scale in which they were recorded [32].

Step 2. Grey relational coefficient (GRC):

The GRC expresses the relation between the best experimental result and the remainder of the experimental values for each variable [33].

By applying Deng's equation, we obtain [34]:

$$
\xi_{i, j}=\frac{\min _{i, j}\left(x_{i, 0}-x_{i, j}\right)+\zeta \max _{i, j}\left(x_{i, 0}-x_{i, j}\right)}{\left(x_{i, 0}-x_{i, j}\right)+\zeta \max _{i, j}\left(x_{i, 0}-x_{i, j}\right)} ; i=1, \ldots, I ; j=1, \ldots, J
$$

where $\zeta$ is the distinguishing coefficient and $\zeta \in[0,1]$. The role of the distinguishing coefficient is to expand or compress the range of the GRC [18]. Usually, $\zeta=0.5$ [35]. $x_{i, 0}$ is the ideal standardised result of the $i$-th variable such that $x_{i, 0} \geq x_{i, j} ; j=1, \ldots \mathrm{J}$.

Step 3. Grey relational grade (GRG):

The GRG is a measure of the GRC values for each variable [36]:

$$
\gamma_{j}=\frac{1}{I} \sum_{i=1}^{I} \xi_{i, j} ; j=1, \ldots, J
$$

The GRG specifies the degree of similarity between the reference sequence (maximum or minimum) and the compared sequence [37].

Step 4. Grey relational rank:

Once the GRGs are estimated, they are arranged in increasing order to form a GRG rank. The higher is the GRG, the better is the option.

\subsubsection{GRGe}

The uncertainty is a parameter that determines the extent to which the result of a measurement represents the real value of the measured quantity [38]. Thus, an experimental result would be incomplete if it is not accompanied by a quantitative and valid expression of its uncertainty. Moreover, after evaluating all of the error components and correcting the analysis, there will always 
be an uncertainty in the accuracy of the result [39]. Unfortunately, most of the experimental results in the field of biomass combustion are expressed without their associated uncertainties, leading to incomplete statements. Additionally, the experimental results on the biomass combustion on a fixed bed are particular in that the factors are interrelated. Thus, the uncertainty of the combined value is a function of the uncertainties of the constituent terms [39].

To quantify the uncertainty during the experiments, each experimental point was obtained from several measurements [22]. The uncertainties that were related to the measurement processes have been estimated for the thermocouple positions and the data acquisition time. With regard to the type-K thermocouples, the precision with which the connectors were embedded inside the combustion tube was analysed, and an estimated mean position variability of $2.06 \mathrm{~mm}$ was obtained. The data acquisition system has a four-second uncertainty in the measurements (the sampling time). An accurate quantification of the uncertainty in the results is crucial for determining the error propagation in subsequent operations [40].

Although the uncertainty parameter does not affect the calculation of the GRG, it does offer additional information that supports the selection of a particular option [20]. The application of EPT enables the quantification of the effects that input measurement uncertainties have on the operations they are involved with [20].

The GRGe is calculated using the following equation [20]:

$$
\Delta\left(\gamma_{j}\right) \approx+\sqrt{\sum_{i=1}^{I} \frac{1}{I^{2}} \sigma^{2}\left(\xi_{i, j}\right)+2 \sum_{i=1}^{I-1} \sum_{k=i+1}^{I} \frac{1}{I^{2}} \sigma\left(\xi_{i, j}\right) \sigma\left(\xi_{k, j}\right) \rho\left(\xi_{i, j}, \xi_{k, j}\right)}
$$

where $\sigma\left(\xi_{i, j}\right)$ represents the uncertainty that is associated with $\xi_{i, j}$, which is a function of the initial uncertainty error of the measurements, thus enabling the quantification of the propagation of the initial uncertainty in the sequence of GRA operations; $\rho\left(\xi_{i, j}, \xi_{k, j}\right)$ is the correlation coefficient between $\xi_{i, j}$ and $\xi_{k, j}$. This equation for calculating the GRGe was estimated using EPT methodology.

\section{Results and Discussion}

To determine the performance of the fuel combustion process, GRA is applied to determine the optimal level conditions. The-larger-the-better case is desirable for the ignition mass flow, and the-smaller-the-better case is desirable for the maximum temperature. Table 3 summarizes the results of 16 sequences that were obtained from a combination of the ignition mass flow rate and the maximum temperature for a particular air flow rate. These sequences were distributed among the three analyzed fuels as a function of the air flow rates that each fuel can tolerate.

The grey relational grade enables the determination of the optimal combination of the experimental parameters for each fuel and an estimation of the influence of each parameter. However, if the uncertainty that is associated with the experimental measurements is not taken into account, the analysis of the grey relational grade variability could advise against selecting a particular combination. Thus, the uncertainty propagation was estimated using the GRGe equation that was given above. 
Table 3. Experimental results, grey relational grade (GRG), and grey relational grade error (GRGe). AMF: Air mass flow $\left(\mathrm{kg} \mathrm{m}^{-2} \mathrm{~s}^{-1}\right)$; IMF: Ignition mass flux $\left(\mathrm{kg} \mathrm{m}^{-2} \mathrm{~s}^{-1}\right)$; IMFU: Ignition mass flux uncertainty $\left(\mathrm{kg} \mathrm{m}^{-2} \mathrm{~s}^{-1}\right)$; MT: Maximum temperature $\left({ }^{\circ} \mathrm{C}\right)$; MTU: Maximum temperature uncertainty $\left({ }^{\circ} \mathrm{C}\right)$; and AE: Air excess.

\begin{tabular}{|c|c|c|c|c|c|c|c|c|c|c|}
\hline Fuel & AMF & IMF & IMFU & IMFU (\%) & MT & MTU & MTU (\%) & $\mathbf{A E}$ & GRG & GRGe \\
\hline \multirow{6}{*}{$\begin{array}{c}\text { Brassica } \\
\text { pellet (bp) }\end{array}$} & 0.08 & 0.04 & $1.74 \times 10^{-3}$ & 4.69 & 740 & 2.40 & 0.32 & 0.39 & $6.67 \times 10^{-1}$ & 0.00 \\
\hline & 0.15 & 0.05 & $2.40 \times 10^{-3}$ & 4.89 & 910 & 3.20 & 0.35 & 0.49 & $5.44 \times 10^{-1}$ & $2.17 \times 10^{-2}$ \\
\hline & 0.18 & 0.06 & $2.75 \times 10^{-3}$ & 4.50 & 1240 & 9.00 & 0.73 & 0.55 & $5.68 \times 10^{-1}$ & $9.38 \times 10^{-2}$ \\
\hline & 0.23 & 0.06 & $2.93 \times 10^{-3}$ & 4.88 & 1300 & 9.00 & 0.69 & 0.72 & $5.35 \times 10^{-1}$ & $8.64 \times 10^{-2}$ \\
\hline & 0.35 & 0.07 & $4.08 \times 10^{-3}$ & 6.27 & 1290 & 9.00 & 0.70 & 0.97 & $6.69 \times 10^{-1}$ & $2.56 \times 10^{-3}$ \\
\hline & 0.38 & 0.05 & $2.69 \times 10^{-3}$ & 5.18 & 1040 & 4.00 & 0.38 & 1.38 & $5.01 \times 10^{-1}$ & $3.20 \times 10^{-2}$ \\
\hline \multirow{5}{*}{$\begin{array}{c}\text { Olive } \\
\text { stone (os) }\end{array}$} & 0.13 & 0.05 & $2.46 \times 10^{-3}$ & 5.13 & 880 & 3.20 & 0.36 & 0.42 & $6.67 \times 10^{-1}$ & 0.00 \\
\hline & 0.15 & 0.05 & $3.10 \times 10^{-3}$ & 5.96 & 930 & 3.20 & 0.34 & 0.54 & $5.94 \times 10^{-1}$ & $1.27 \times 10^{-1}$ \\
\hline & 0.23 & 0.06 & $2.65 \times 10^{-3}$ & 4.81 & 1010 & 0.00 & 0.00 & 0.66 & $6.29 \times 10^{-1}$ & $3.21 \times 10^{-1}$ \\
\hline & 0.28 & 0.05 & $2.34 \times 10^{-3}$ & 4.59 & 1100 & 4.00 & 0.36 & 0.87 & $3.89 \times 10^{-1}$ & $8.11 \times 10^{-2}$ \\
\hline & 0.35 & 0.06 & $4.58 \times 10^{-3}$ & 8.17 & 960 & 3.20 & 0.33 & 0.85 & $7.89 \times 10^{-1}$ & $6.19 \times 10^{-3}$ \\
\hline \multirow{5}{*}{$\begin{array}{c}\text { Wood } \\
\text { pellet (wp) }\end{array}$} & 0.15 & 0.05 & $4.21 \times 10^{-3}$ & 8.42 & 890 & 3.20 & 0.36 & 0.43 & $6.67 \times 10^{-1}$ & 0.00 \\
\hline & 0.33 & 0.07 & $4.18 \times 10^{-3}$ & 6.06 & 1020 & 4.00 & 0.39 & 0.81 & $7.23 \times 10^{-1}$ & $4.62 \times 10^{-3}$ \\
\hline & 0.35 & 0.07 & $6.12 \times 10^{-3}$ & 9.14 & 990 & 3.20 & 0.32 & 0.98 & $6.69 \times 10^{-1}$ & $2.58 \times 10^{-1}$ \\
\hline & 0.43 & 0.06 & $6.31 \times 10^{-3}$ & 10.35 & 1040 & 4.00 & 0.38 & 1.21 & $4.77 \times 10^{-1}$ & $1.08 \times 10^{-1}$ \\
\hline & 0.48 & 0.05 & $4.30 \times 10^{-3}$ & 8.43 & 1100 & 4.00 & 0.36 & 1.63 & $3.39 \times 10^{-1}$ & $3.69 \times 10^{-2}$ \\
\hline
\end{tabular}

Once the GRG and GRGe are calculated for each data sequence, the results are plotted (Figures 6-8, right-hand side). The order of the sequence that is shown in the GRG rank plots (right area of the plots) matches the one that was used to represent the effect of the air mass flow on the ignition mass flow and on the temperature in the ignition front (left area of the plots).

The figures show that, except for the wp (Figure 7), the highest GRG values have associated GRGe values that are small enough to ensure that these GRG values generally correspond to the best options. In the case of the wp, $\mathrm{GRG}_{2}$ has such a high GRGe value because the ignition mass flow for this sequence has an associated uncertainty of $9.14 \%$ for the ignition mass flow. This uncertainty propagates along all of the operation sequence, resulting in a GRGe value of $2.58 \times 10^{-1}$, which represents $38.60 \%$ of the $\mathrm{GRG}_{2}$ value.

Figure 6 presents the effect of the air mass flow on the ignition mass flow for the bp. The behavior of the bp (Figure 6) serves as a generic model for the other fuels that are under consideration. Although the air flow rates and velocities differ, three combustion phases are distinguishable for all of the fuels as a function of the supplied air mass flow [41]. The first phase corresponds to a linear increase in the combusted mass flow (the oxygen-limited phase), followed by a quasi-constant speed zone (the reaction-limited phase), followed by the third and last quenching phase (the convective cooling phase). These three phases are not as clearly distinguishable in the other fuels because of the lower flow rates. A continuous slanted line divides this figure into two zones and represents the fuel stoichiometric line. The measurements that correspond to the left-hand side of this line belong to the sub-stoichiometric regime, while the measurements on the right involve an excess of air. The fact that the stoichiometric regime was estimated for the whole fuel and not for the char must be taken into account. 
The tests that were performed with the bp exhibited the best combination of air flow, ignition mass flow, and maximum temperature; this combination is reached at the point of maximum ignition flow. The second best combination $\left(\mathrm{GRG}_{1}\right)$ is obtained with the smallest possible maximum temperature, which corresponds to the sequence that has the lowest air flow rate, $0.075\left(\mathrm{~kg} \mathrm{~m}^{-2} \mathrm{~s}^{-1}\right)$. As observed in Figure 6, these two configurations clearly exhibit the best results. Taking the GRGe values into account, both options could be considered optimal. The excess air value in $\mathrm{GRG}_{5}$ is very close to the stoichiometric value, which is related to the fact that such a point falls within the zone that corresponds to the reaction-limited mode, in which the ignition mass flow is a function of the rate of heat transfer into the fuel particles.

Figure 6. Air mass flow rate effect on the ignition mass flow rate and the front temperature of the brassica pellet (bp), and the GRG rank.

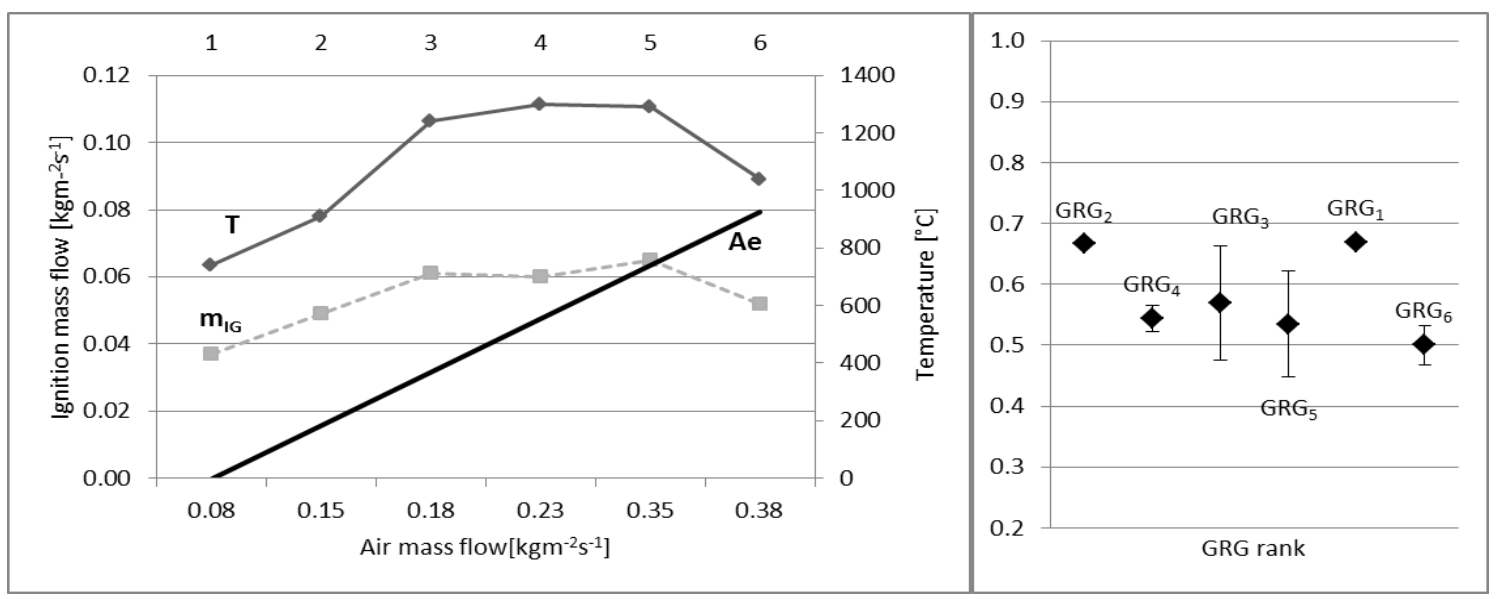

In the case of the wp (Figure 7), the best option is $\mathrm{GRG}_{2}$, which is the test with the highest ignition mass flow; however, the temperature does not correspond to the smallest maximum temperature value, which was the other purpose of this test. This result could be due to the relation that exists between the temperature that was reached in the fixed bed and the propagation speed. The higher is the reaction temperature, the higher is the combusted fuel mass flow, and vice versa [14]. There are two distinguishable zones in Figure 7 (GRG ranks), which differ as a function of the excess air; the first three combinations (the sub-stoichiometric zone) are clearly better than the last two. This result arises because all of the maximum rates are reached under fuel-rich conditions, and therefore, the optimal condition point varies as a function of the utilized fuel. The combination that is represented by $\mathrm{GRG}_{2}$ is not recommended because of its high GRGe value. The configuration $\mathrm{GRG}_{3}$ is more suitable, although it has a lower GRG value. A comparison of the $\mathrm{GRG}_{2}$ and $\mathrm{GRG}_{1}$ sequences shows that these sequences correspond to two distinct combustion regimes (see Figure 7). $\mathrm{GRG}_{2}$ is located in the reaction-limited phase, in which the ignition rate is limited by the fuel combustion rate. GRG $_{1}$ is located in the oxygen-limited phase, in which the propagation front is affected by the volatiles, which need to react with the char when the oxygen content is deficient. These sequences are the best two options due to the effect of the fuel properties on the fixed bed morphology. The small size of the wp particles cause the fixed bed to acquire a high porosity during the initial combustion phase (oxygen-limited mode). Thus, enhancing the air mass flow from the bed base to the higher layers at which combustion occurs. 
Figure 7. Air mass flow rate effect on the ignition mass flow rate and the front temperature of the wood pellets (wp), and the GRG rank.

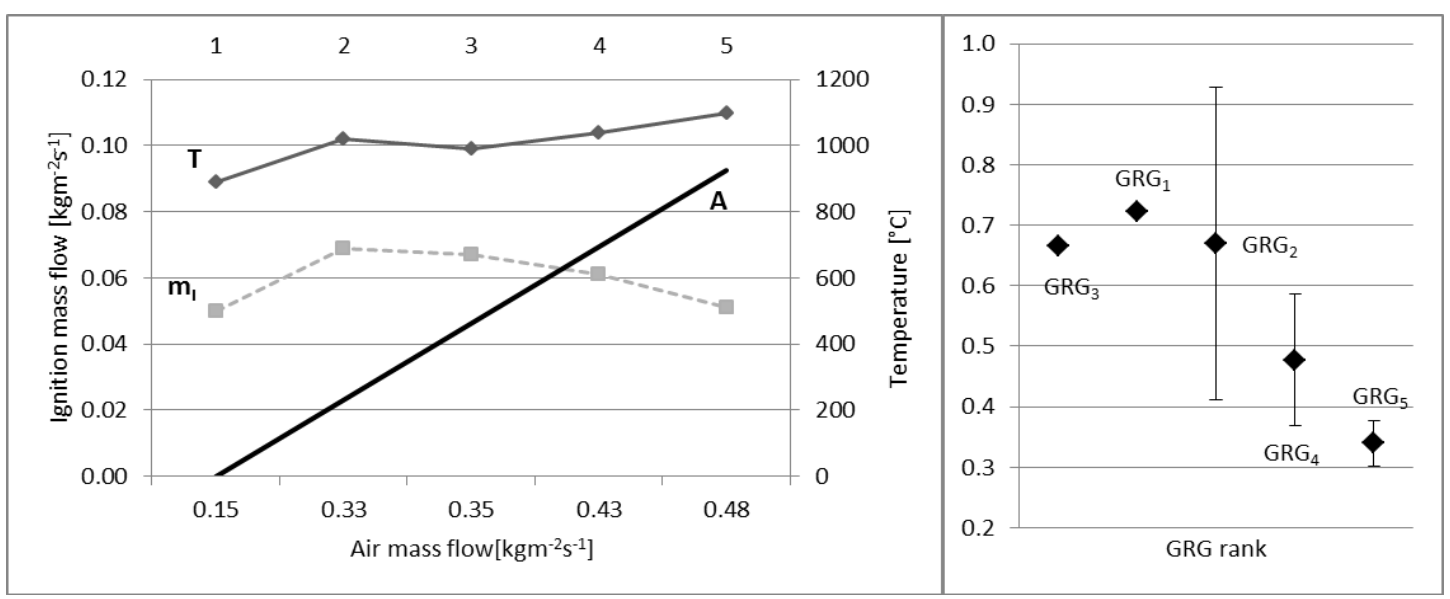

Figure 8 corresponds to the utilisation of the os; here, as in the case of the wp, $\mathrm{GRG}_{5}$ is a combination of the maximum ignition flow $\left(\dot{m}_{i g-\max }^{\prime \prime}\right)$ and a maximum temperature above the minimum. The ignition mass flow rate for $\mathrm{GRG}_{5}$ is $0.056\left(\mathrm{~kg} \mathrm{~m}^{-2} \mathrm{~s}^{-1}\right)$, which is the lowest $\dot{m}_{i g-\text { max }}^{\prime \prime}$ value of the three fuels that were tested. This fact indicates that there is a relation between the fuel moisture content and the maximum propagation speed that was reached. Thus, the os, which has the highest moisture content (13\%), had the lowest maximum propagation speed. However, the os moisture content has no effect on the maximum temperature. Although the effect of the moisture content has been sufficiently verified $[24,29,42]$, the results warn against the generalization of such an effect on low moisture content fuels. Along the same line, several studies $[43,44]$ have found that an increase in the moisture content decreases the ignition front propagation speed due to the energy that is consumed by the evaporation process, although no drastic changes in the reached temperature were observed up to a moisture content of greater than $20 \%$.

Figure 8. Air mass flow rate effect on the ignition mass flow rate and the front temperature of the olive stone (os), and the GRG rank.

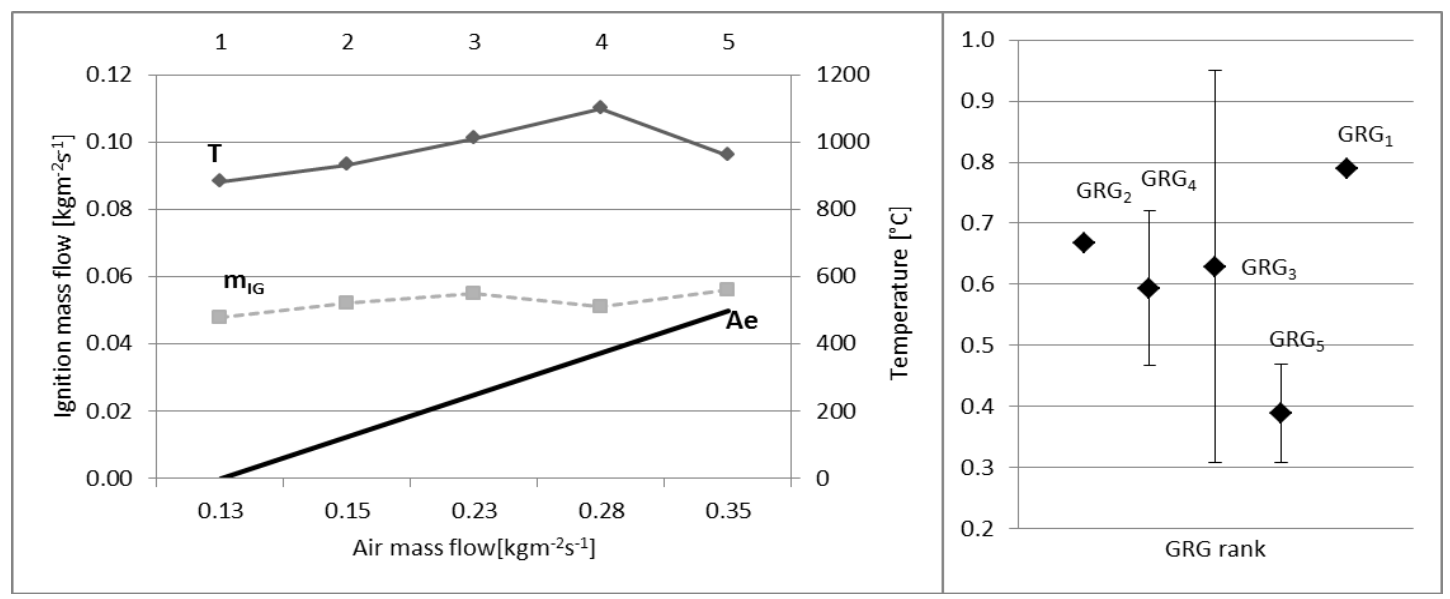

A fact that should be highlighted is that, for every fuel, the best options correspond to conditions in the sub-stoichiometric zone, i.e., the excess air is below 1 (the combustion/gasification zone with small 
$\mathrm{NO}_{x}$ formation). As expected, the worst options are related to conditions that have an excess air of above 1. The GRG rank shows two distinguishable zones (Figures 6 and 7) that are located in the upper section of the sub-stoichiometric regime because the maximum rates are reached under fuel-rich conditions. This fact indicates the adverse effect of convection in counter-current combustion fixed beds. The optimal condition will vary as a function of the type of fuel that is being analysed. Moreover, there is a broader range of stable operations in sub-stoichiometric regimes.

It was observed that the best combinations correspond to the conditions in which $\dot{m}_{i g-\max }^{\prime \prime}$ is reached. If a specific analysis is performed for each of the conditions that have a maximum ignition rate, the results indicate that this value decreases as it nears the stoichiometric condition, which is a consequence of the adverse effect of convection. The higher is the air requirement to reach the maximum, the higher is the heat that is lost by convection and the lower the rate and the energy that is supplied. By comparing the global rates (Table 3), it can be seen that the maximum value is reached by the wp $\left(0.069 \mathrm{~kg} \mathrm{~m}^{-2} \mathrm{~s}^{-1}\right)$, which also have the broadest operating range when their combustion is conducted in a zone close to a $0.5 \mathrm{~kg} \mathrm{~m}^{-2} \mathrm{~s}^{-1}$ air mass flow. However, the os is the fuel that first reaches the extinction convection zone with an air flow of $\left(0.352 \mathrm{~kg} \mathrm{~m}^{-2} \mathrm{~s}^{-1}\right)$. There is a clear relation between the lower heating value (LHV) and the ignition front propagation speed. Thus, the best quality fuels (with higher LHV) reach higher ignition flow rate values [24].

Although the graphical representations of the maximum temperature and the ignition mass flow as a function of air flow (left area of plots) present information on the points that correspond to the best possible conditions, they do not take into account the individual and/or combined effect of the measured variable uncertainty. Thus, for the wp (Figure 7), condition three could be considered the second best combination of values; however, the high GRGe value that corresponds to condition three invalidates this option. As expected, the selection of the optimal condition becomes more complex as more variables are taken into account; in this sense, GRA offers a simplification of the decision making when selecting the best combustion conditions. Moreover, the GRG and GRGe structured method avoids leaving the selection criteria to the observer, providing more valid results.

\section{Conclusions}

This study analyzed the behavior of three fuels in a counter-current fixed bed reactor, and the best combustion configuration was selected using a combination of EPT and GRA, while not considering combustions aspects.

The results showed that the fuel composition and the morphology, in terms of size, are determinant factors for reaching the maximum propagation rate of the ignition front. The maximum ignition mass flow values are reached by the wp, which is the fuel with the smallest particle size, resulting in a higher heat transfer between the different bed layers. On the contrary, the os exhibited the lowest maximum ignition mass flow rate because of its high water content. All of the fuel optimal combinations belong to the sub-stoichiometric regime, indicating the importance of the convection adverse effect on counter-current combustion fixed beds.

The distinction among the sub-stoichiometric regime and the excess air regime is shown by the GRG rank, in which two zones of value ranges can be observed. This phenomenon is a result of 
reaching the maximum rates under fuel-rich conditions. The point that corresponds to the optimal condition varies as a function of the analyzed fuel.

\section{Acknowledgments}

The authors acknowledge financial support from the project 10DPI303006PR and CN2012/249 of the Xunta de Galicia. The work of Angeles Saavedra was supported by Spanish Ministry of Science and innovation projects MTM2008-03129 and MTM2011-23204.

\section{Conflicts of Interest}

The authors declare no conflict of interest.

\section{References}

1. The Spanish Ministry of Industry, Energy and Tourism. Instituto para la Diversificación y Ahorro de la Energía (IDEA). Plan de Acción de Ahorro y Eficiencia Energética 2011-2020 (in Spanish). Available online: http://www.idae.es/index.php/mod.documentos/mem.descarga?file=/documentos 11905_PAEE_2011_2020._A2011_A_a1 e6383b.pdf (accessed on 13 September 2013).

2. Directorate General for Energy. EU Energy Trends to 2030. Available online: http:/ec. europa.eu/energy/observatory/trends_2030/doc/trends_to_2030_update_2009.pdf (accessed on 13 September 2013).

3. Nussbaumer, T. Combustion and co-combustion of biomass: Fundamentals, technologies, and primary measures for emission reduction. Energy Fuels 2003, 17, 1510-1521.

4. Senneca, O. Kinetics of pyrolysis, combustion and gasification of three biomass fuels. Fuel Process. Technol. 2007, 88, 87-97.

5. Chaney, J.; Liu, H.; Li, J. An overview of CFD modelling of small-scale fixed-bed biomass pellet boilers with preliminary results from a simplified approach. Energy Convers. Manag. 2012, 63, 149-156.

6. Collazo, J.; Pazo, J.A.; Granada, E.; Saavedra, A.; Eguia, P. Determination of the specific heat of biomass materials and the combustion energy of coke by DSC analysis. Energy 2012, 45, 746-752.

7. Mehrabian, R.; Scharler, R.; Obernberger, I. Effects of pyrolysis conditions on the heating rate in biomass particles and applicability of TGA kinetic parameters in particle thermal conversion modelling. Fuel 2012, 93, 567-575.

8. Wang, T.; Yang, H.; Wu, Y.; Liu, Q.; Lv, J.; Zhang, H. Experimental study on the effects of chemical and mineral components on the attrition characteristics of coal ashes for fluidized bed boilers. Energy Fuels 2012, 26, 990-994.

9. Anca-Couce, A.; Zobel, N.; Jakobsen, H.A. Multi-scale modeling of fixed-bed thermo-chemical processes of biomass with the representative particle model: Application to pyrolysis. Fuel 2013, 103, 773-782.

10. Houshfar, E.; Lovas, T.; Skreiberg, O. Experimental investigation on $\mathrm{NO}_{x}$ reduction by primary measures in biomass combustion: Straw, peat, sewage sludge, forest residues and wood pellets. Energies 2012, 5, 270-290. 
11. Morissette, R.; Savoie, P.; Villeneuve, J. Combustion of corn stover bales in a small 146-kW boiler. Energies 2011, 4, 1102-1111.

12. Perez, J.F.; Melgar, A.; Benjumea, P.N. Effect of operating and design parameters on the gasification/combustion process of waste biomass in fixed bed downdraft reactors: An experimental study. Fuel 2012, 96, 487-496.

13. Horttanainen, M.; Saastamoinen, J.; Sarkomaa, P. Operational limits of ignition front propagation against airflow in packed beds of different wood fuels. Energy Fuels 2002, 16, 676-686.

14. Johansson, R.; Thunman, H.; Leckner, B. Sensitivity analysis of a fixed bed combustion model. Energy Fuels 2007, 21, 1493-1503.

15. Liu, S.; Lin, Y. Grey Systems: Theory and Applications; Springer-Verlag: Berlin, Germany, 2010.

16. Wang, H.; Jiang, X.; Liu, J.; Lin, W. Experiment and grey relational analysis of CWS spheres combustion in a fluidized bed. Energy Fuels 2007, 21, 1924-1930.

17. Asian, N.; Shahrivar, A.A.; Abdollahi, H. Multi-objective optimization of some process parameters of a lab-scale thickener using grey relational analysis. Sep. Purif. Technol. 2012, 90, 189-195.

18. Kuo, Y.; Yang, T.; Huang, G. The use of grey relational analysis in solving multiple attribute decision-making problems. Comput. Ind. Eng. 2008, 55, 80-93.

19. Yin, C.; Rosendahl, L.; Clausen, S.; Hvid, S.L. Characterizing and modeling of an $88 \mathrm{MW}$ grate-fired boiler burning wheat straw: Experience and lessons. Energy 2012, 41, 473-482.

20. Saavedra, A.; Arce, E.; Miguez, J.L.; Granada, E. Potential effect of uncertainty on the GRG interpretation. Grey Syst. Theory Appl. 2013, 3, 121-128.

21. Joint Committee for Guides in Metrology (JCGM). International Vocabulary of Metrology-Basic and General Concepts and Associated Terms (VIM), 2012. Available online: http://www.bipm.org/ utils/common/documents/jcgm/JCGM_200_2012.pdf (accessed on 13 September 2013).

22. Porteiro, J.; Patiño, D.; Collazo, J.; Granada, E.; Moran, J.; Miguez, J.L. Experimental analysis of the ignition front propagation of several biomass fuels in a fixed-bed combustor. Fuel $\mathbf{2 0 1 0}$, $89,26-35$.

23. Patino, D. Análisis Experimental de Combustión de Biomasa en un Quemador de Lecho Fijo (in Spanish). Ph.D. Thesis, University of Vigo, Vigo, Spain, 2009.

24. Shin, D.; Choi, S. The combustion of simulated waste particles in a fixed bed. Combust. Flame 2000, 121, 167-180.

25. Hermansson, S.; Thunman, H. Measures to reduce grate material wear in fixed-bed combustion. Energy Fuels 2011, 25, 1387-1395.

26. Thunman, H.; Leckner, B. Co-current and counter-current fixed bed combustion of biofuel-A comparison. Fuel 2003, 82, 275-283.

27. Porteiro, J.; Patiño, D.; Miguez, J.L.; Granada, E.; Moran, J.; Collazo, J. Study of the reaction front thickness in a counter-current fixed-bed combustor of a pelletised biomass. Combust. Flame 2012, 159, 1296-1302.

28. Buczynski, R.; Weber, R.; Szlek, A.; Nosek, R. Time-dependent combustion of solid fuels in a fixed-bed: Measurements and mathematical modeling. Energy Fuels 2012, 26, 4767-4774.

29. Bidabadi, M.; Abedinejad, M.S.; Fereidooni, J. Modeling of the propagation of a reaction front in fixed bed combustion of wood particles. J. Mech. 2011, 27, 453-459. 
30. Bitra, V.S.P.; Womac, A.R.; Yang, Y.T.; Igathinathane, C.; Miu, P.I.; Chevanan, N.; Sokhansanj, S. Knife mill operating factors effect on switchgrass particle size distributions. Bioresour. Technol. 2009, 100, 5176-5188.

31. Liao, K.; Yao, Q.; Wu, X.; Jia, W. A numerical corrosion rate prediction method for direct assessment of wet gas gathering pipelines internal corrosion. Energies 2012, 5, 3892-3907.

32. Moran, J.; Granada, E.; Míguez, J.L.; Porteiro, J. Use of grey relational analysis to assess and optimize small biomass boilers. Fuel Process. Technol. 2006, 87, 123-127.

33. Subbaya, K.M.; Suresha, B.; Rajendra, N.; Varadarajan, Y.S. Grey-based Taguchi approach for wear assessment of SiC filled carbon-epoxy composites. Mater. Des. 2012, 41, 124-130.

34. Deng, J. Control problems of grey systems. Syst. Control Lett. 1982, 1, 288-294.

35. Chang, A.Y. Prioritising the types of manufacturing flexibility in an uncertain environment. Int. J. Prod. Res. 2012, 50, 2133-2149.

36. Li, H.Z.; Tao, W.; Gao, T.; Li, H.; Lu, Y.H.; Su, Z.M. Improving the accuracy of density functional theory (DFT) calculation for homolysis bond dissociation energies of Y-NO bond: Generalized regression neural network based on grey relational analysis and principal component analysis. Int. J. Mol. Sci. 2011, 12, 2242-2261.

37. Lee, W.; Lin, Y. Evaluating and ranking energy performance of office buildings using grey relational analysis. Energy 2011, 36, 2551-2556.

38. Joint Committee for Guides in Metrology (JCGM). Evaluation of Measurement Data - Guide to the Expression of Uncertainty in Measurement, September 2008. Available online: http:/www.bipm.org/ utils/common/documents/jcgm/JCGM_100_2008_E.pdf (accessed on 13 September 2013).

39. Hughes, I.G.; Hase, T.P.A. Measurements and Their Uncertainties: A Practical Guide to Modern Error Analysis; Oxford University Press: Oxford, UK, 2010.

40. Pazo, J.A.; Granada, E.; Saavedra, A.; Patino, D.; Collazo, J. Heterogenic solid biofuel sampling methodology and uncertainty associated with prompt analysis. Int. J. Mol. Sci. 2010, 11, 2118-2133.

41. Ryu, C.; Yang, Y.B.; Khor, A.; Yates, N.E.; Sharifi, V.N.; Switenbank, J. Effect of fuel properties on biomass combustion: Part I. Experiments-Fuel type equivalence ratio and particle size. Fuel 2006, 85, 1039-1046.

42. Saastamoinen, J.J.; Taipale, R.; Horttanainen, M.; Sarkomaa, P. Propagation of the ignition front in beds of wood particles. Combust. Flame 2000, 123, 214-226.

43. Liang, L.; Sun, R.; Fei, J.; Wu, S.; Liu, X.; Dai, K.; Yao, N. Experimental study on effects of moisture content on combustion characteristics of simulated municipal solid wastes in a fixed bed. Bioresour. Technol. 2008, 99, 7238-7246.

44. Zhao, W.; Li, Z.; Zhao, G.; Zhang, F.; Zhu, Q. Effect of air preheating and fuel moisture on combustion characteristics of corn straw in a fixed bed. Energy Convers. Manag. 2008, 49, 3560-3565.

(C) 2013 by the authors; licensee MDPI, Basel, Switzerland. This article is an open access article distributed under the terms and conditions of the Creative Commons Attribution license (http://creativecommons.org/licenses/by/3.0/). 